\title{
Comparison of Tungsten Carbide and Arkansas Stone For Adhesive Removal On Enamel Surface After Debonding Procedure
}

\author{
Aditya Kartika Noviyanti Putri, Thalca Hamid, Ari Triwardhani \\ Department of Orthodontics, Faculty of Dental Medicine \\ Universitas Airlangga \\ Surabaya - Indonesia
}

\begin{abstract}
Background: Debonding aims to remove orthodontic appliances from the patient's teeth and cleanse all residual adhesive material on the surface of the tooth to restore surface conditions such as before orthodontic treatment. Damage may occur after debonding process. Removal of the remaining adhesive material has a major effect on the enamel surface conditions, the researcher wants to know the effect of cleaning adhesive material using Tungsten carbide 12 flute burs, Arkansas stone bur on the enamel surface Purpose: To determine the effect of cleaning the remaining adhesive material using Tungsten carbide 12 flute burs and Arkansas stone bur Against the Surface of the Enamel. Method: This study was experimental laboratory (in vitro study) with 4 groups division, first control group was tooth before bracket was installed, group 2 was done debonding without cleaning of residual adhesive, group 3 was done adhesive cleansing with Arkansas stone and group 4 was done adhesive cleaning with tungsten carbide 12-flutted Result: Showing that the use of 12-flutted tungsten carbide was able to clean enamel surfaces with the most delicate results and did not cause cracks or cracks on enamel surface Conclusions: The best method to clean the remaining adhesive is using 12-flutted tungsten carbide bur using contra angle high speed and with adequate water flow and sprays to prevent increasing of temperature that damage dental pup.
\end{abstract}

Keywords: Debonding, Adhesive removal and surface roughness

Correspondence: Thalca Hamid, Department of Orthodontics, Faculty of Dental Medicine, Universitas Airlangga, J1. Prof. Dr. Moestopo 47 Surabaya 60132 - Indonesia, Phone: 031-5030255 Email: thalca@fkg.unair.ac.id

\section{INTRODUCTION}

Debonding aims to remove orthodontic appliance from the patient's teeth and clean all the remaining adhesive materials that remained on tooth surface to restore the surface condition of as clean as possible before orthodontic treatment ${ }^{1}$. The attachment of adhesive materials used acid etching, etching results in microporosity of the enamel surface so that microretention occurred from resin that infiltrate into enamel surface. Cleaning the remaining adhesive material mechanically after the debonding process is very detrimental to enamel because it causes the enamel layer to erode significantly and the damage is irreversible ${ }^{1,2}$.

Many patients complain that their teeth feel rough because the rest of the adhesive material was not clean completely and the remnants of adhesive material tend to change color as time goes by ${ }^{3}$. Cleaned the remaining adhesive can be done in several ways including by scraping and using bur techniques. Removing the bracket and cleaning the remaining adhesive materials must be carried out carefully and minimizing the occurrence of the damage to enamel layer. The outer layer of enamel is a layer that is rich in fluoride ${ }^{4,5}$. Damage that may occur after debonding procedure, such as rough enamel surfaces, vertical cracks and pulp necrosis. The appearance of rough areas on the enamel surface or cracks on the surface of teeth can cause enamel discoloration and plaque accumulation which causes enamel demineralization and reduce aesthetic values ${ }^{3}$. Damage to the enamel surface after debonding is unavoidable, but can be minimized if the clinician debonds with appropriate techniques ${ }^{6}$.

As mentioned above, there are two ways to clean adhesive residue after debonding, first is using scrapping technique with adhesive remover pliers and second with certain bur such as tungsten carbide on low speed or high speed, use shofu bur, Sof-Lex ${ }^{\circledR}$ disc, fiberglass burs. Some studies said that the use of handpieces has proven to produce the most satisfying results, but some studies have found that they are more damaging than green rubber wheels ${ }^{7}$.

Various types of research have not shown specific results regarding the most efficient and safe way to clean the remaining adhesive material, considering the devices mentioned above are often used by orthodontists. Based on 
the description described above, cleaning the residual adhesive material has a major influence on the enamel surface conditions, various cleaning methods still need to be investigated to minimize enamel surface damage, so as to be able to restore the tooth surface color and condition as well as before orthodontic treatment. The study was aimed to know the effect of cleaning the adhesive material using tungsten carbide bur and Arkansas stone bur on the enamel surface.

\section{METHODS \\ Study Design}

It was an experimental study with a post-test only in control group design to compare Tungsten carbide and Arkansas stone for removing adhesive on enamel surface after debonding procedure. The total number of samples in this study were 20 samples, in which 20 premolars samples were divided into 4 groups, namely control group: consists of 5 dental samples that were not treated at all after the 2 day incubation period as a comparison to other groups., group 1: consists of 5 samples of premolar which was placed a bracket after 2 days incubation period, after bracket was placed, teeth were incubated for 2 days and debonding bracket carried out without cleaning the remaining adhesive material, group 2: consists of 5 samples of premolars which was placed a bracket after 2 days incubation period, after bracket was placed, teeth were incubated for 2 days and debonding bracket is carried out. The remaining adhesive materials were cleaned by contra angle high speed with the Arkansas stone bur for 30 seconds, group 3: consists of 5 samples of premolars which was placed a bracket after 2 days incubation period, after bracket was placed, teeth were incubated for 2 days and debonding bracket is carried out. The remaining adhesive materials were cleaned by contra angle high speed with Tungsten carbide bur 12 flute for 30 seconds.

\section{Surface Roughness}

Enamel's surface roughness was evaluated used scanning electron microscope (SEM) by comparing the control group with another group. In addition, the number of cracks and the length of the cracks were evaluated.
Assessment of surface roughness also used enamel surface index (ESI) by Zacharisson and Arthun (1980) with criteria ${ }^{8}$ : Score 0: Perfect surface conditions. No cratches, intact enamel perikymata; score 1: Good surface conditions, but there are fine scratches or lines of some perikymata; score 2: Surface conditions are still acceptable. There are scratches more and some deeper scratches, none perikymata; score 3: imperfect surface conditions. There are a few scratches varying in depth and rough, there is no perikymata; score 4: Surface condition isn't acceptable. Rough scratches, very deep and destructive

\section{Bracket Placement and Debonding Procedure}

The buccal surface of the tooth was rinsed under tap water and dried, then marked the area to be etched then the enamel surface that had been prepared is etched and wait for 50 seconds. Next, rinsed using three-way syringe for 10 seconds and dried with three-way syringe spray and cover the enamel surface and mesh bracket with thin liquid bonding. Adhesive paste was applied about $1 \mathrm{~mm}$ on the bracket surface, then placed bracket on the surface of the teeth by being pressed from the gingiva to occlusal, excess adhesive is taken with sonde. The tooth that had been attached to the bracket was inserted into the container and given artificial saliva solution, then stored in the incubator for 48 hours. Debonding procedure used bracket remover plier with peeling technique

\section{Statistics}

Analysis of enamel surface used scanning electrons microscope (SEM) and assessment of enamel surface roughness using visual comparison of SEM between groups, enamel surface index (ESI) and calculated the number of cracks and the length of cracks on the surface of the tooth. After that the data is tested used Kolmogorov-Smirnov test to check that data was normal distributed, after that, it was followed by a parametric test with one- way ANOVA. For knowing the differences between groups of data were tested descriptively by LSD test and Schefe. 

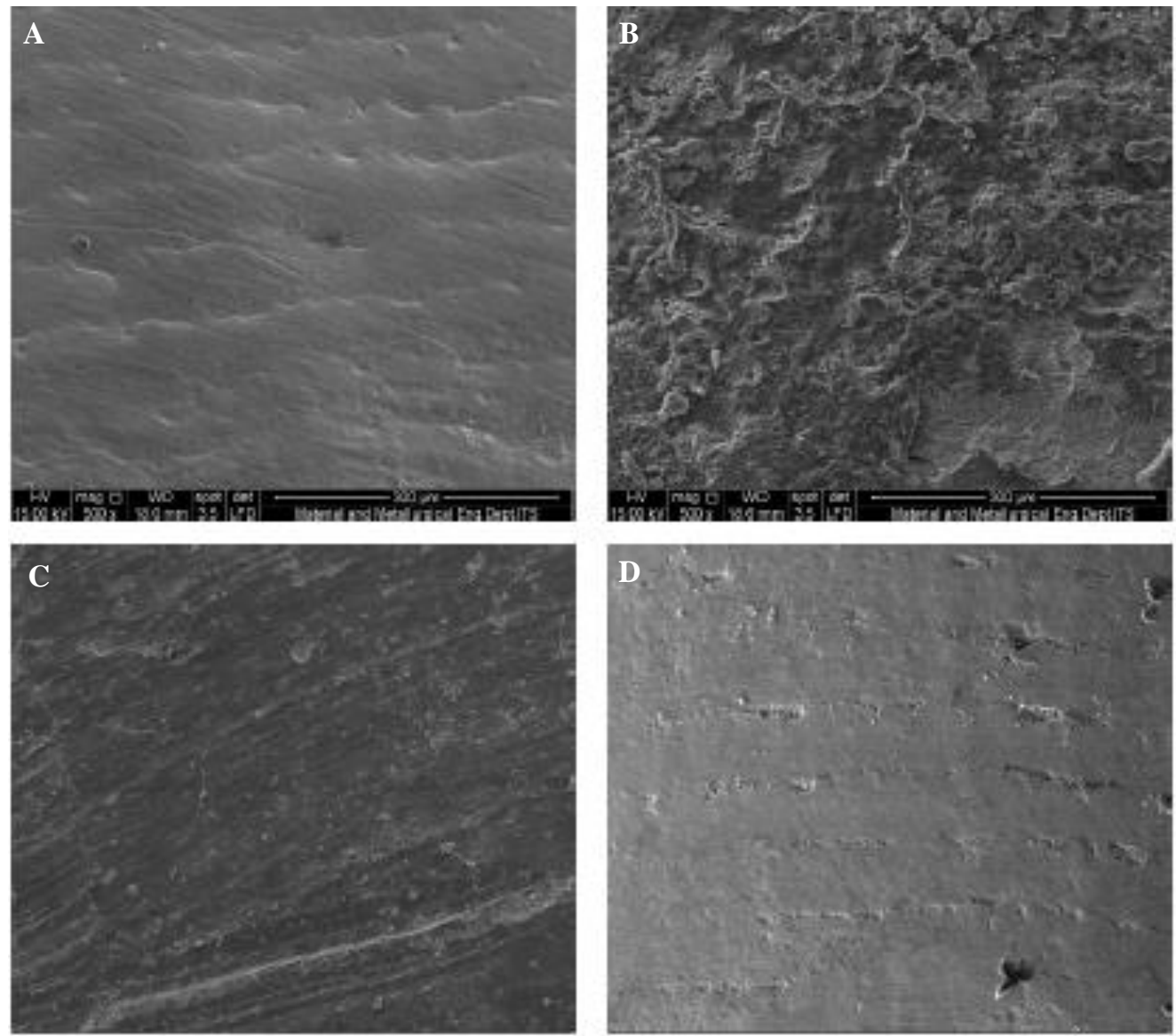

Figure 1. Comparison of the four treatment groups. (A) Control group, (B) Group 1, (C) Group 2 and (D) group 3 in 500x magnification used scanning electron microscope

\section{RESULTS}

\section{Visual Evaluation with SEM}

Visual evaluation of this study used SEM to see the differences in enamel surface of each group that has been given treatment according to the criteria of each group. Evaluation used SEM was possible because it is capable to do 10,000x magnification. SEM utilized electron conductivity on the surface of the sample that already coated by palladium and gold. Coating using palladium and gold were needed to improve conductivity to obtain good surface detail even at the highest magnification level. The study used 50x, 100x, 500x and 1000x magnification. Researcher used SEM to shows a clear difference from each group.

Evaluation of the enamel surface of the control group showed a smooth surface of enamel without cracking and intact perykimata enamel arrangement, with regular distance between one perykimata enamel and the other perykimata enamel. There are no scratches or fine lines. The results of this control group would be compared with another group that received treatment.
Figure 1 explain an evaluation used an electron microscope with 50x magnification compared control groups that were not treated at all with group 1 being that did not get adhesive residual cleaning showed and Figure 2 showed the remaining pile of adhesive material left intact on the enamel surface, there are 3 types areas were $\mathrm{E}, \mathrm{F}$ and $\mathrm{G}$. the area showed a different picture. Area $\mathrm{E}$ was the area that covered by bracket mesh.

This area showed an irregular shape of the base of mesh bracket that had varied depth. The brighter area showed high area compared to the darker areas. Area E was an excessive residue area which located Irregular adhesive material and clear limits. Area F also called an interface between treated tooth surface and covered area. Area $\mathrm{G}$ is an area that was not treated, there were black spots, which is indicated the microporosity of acid etch during preparation of the bracket placement. In addition, it appears microscopic debris of material that cover tooth surface that indicated residual debris as a result of debonding procedure. Figure 1.B shows that debonding without cleaning the remaining adhesive material will damage the enamel 


\section{Indonesian Journal of Dental Medicine}

Volume 1 Issue 1 2018; 59-65

surface. Surface enamel becomes rough, irregular and disrupts dental aesthetics.

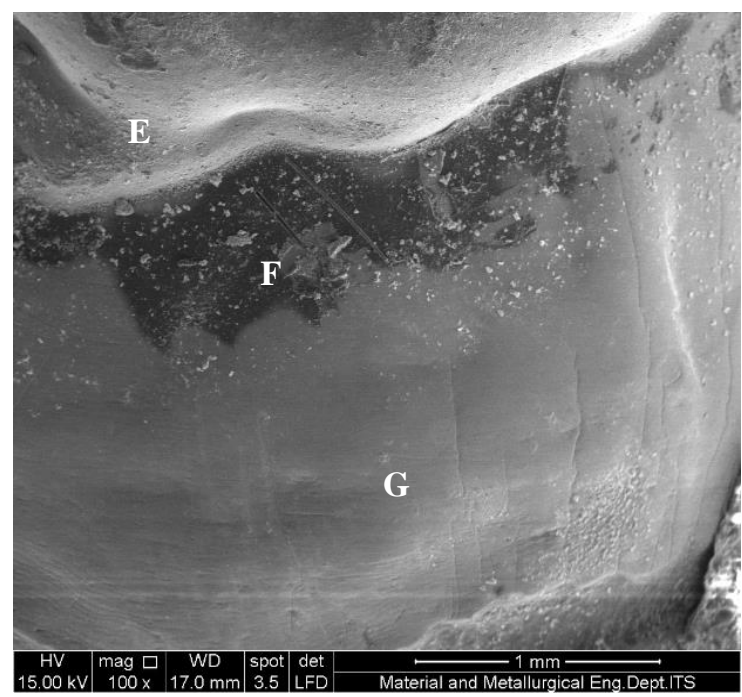

Figure 2. Group 2 with 100x magnification showed (E) was the area that covered by bracket mesh $(\mathrm{F})$ interface between treated tooth surface and covered area, $(\mathrm{G})$ area that was not treated

Group 2 used Arkansas stone bur to clean adhesive materials. Electron microscope image in the sample of this group showed irregular scratches on enamel surface. Figure $1 \mathrm{C}$ showed that the enamel surfaceis rough and there are fine pieces of material on the surface of the tooth. Scratches with various lengths and depths that appear in almost all fields. In addition, there are no intact enamel features. At 500x magnification we get a description of the remaining adhesive material which was still left on the enamel surface. Cleaning with Arkansas stone used high speed contra angle with directional motion, but on SEM observations show that cleaning the residual adhesive material with Arkansas stone leaves scratches with irregular directions and rough surface.

SEM showed erosion with irregular depths in some areas with unidentified debris on the enamel surface. Figure $1 \mathrm{C}$ showed changes in microstructure of enamel surface morphology. In 1C appeared the loss of pericymata enamel, there were scratches and some erosion areas with varying depths. Figure $1 \mathrm{C}$ shows a rough surface, but the degree is lighter if compared to figure 1D. When compared to the surface of control group, it showed that Arkansas stone bur was harm for enamel surface and made rough surface and irregular scratches.

The last group was group 3. Group 3 used 12 flute tungsten carbide. The 5 samples in the group showed a smoother surface when compared to 2 previous group. Some samples have fine lines and loss of pericymata enamel. But tungsten carbide did not scratch with different depths. Some samples are still showed perykimata enamel but not intact. In this group there were no rough scratches or residual debris of adhesive material.

Tungsten carbide was still eroding the enamel surface characterized by the tear of percymata enamel structure and loss of pericymata enamel in some samples. In Figure 1D obtained smooth enamel surface, in 500x magnification shows an enamel surface with a smooth area there are areas that were brighter but uneven, and there is a pore formation on the enamel surface. There are no debris or areas who experience erosion with different depths. Enamel cleansing after the debonding process using a tungsten carbide gave a smooth surface. When compared to Arkansas stone, the use of tungsten carbide 12 flutes produced a smooth enamel surface, and does not leave erosion on the surface.

SEM are able to measure the length of cracks on the enamel surface; the crack length can be analyzed to visually assess the surface condition of the enamel. Group 1 has the lowest score for the length of enamel crack, possibly because some samples in this group were covered by adhesive residues that were not cleaned. The largest crack length was found in group 2, with mean score of 0.79800 and standard deviation of 0.11943 . Group 3 which carried out the cleaning of the residual adhesive 


\section{Indonesian Journal of Dental Medicine}

Volume 1 Issue 1 2018; 59-65

material using tungsten carbide bur has a mean value of 0.39937 and has the largest standard deviation score of 0.29510 . Table 3 and table 4 shows descriptive results the length of the crack using Schefe test and continue with ANOVA. It was known that between groups have significant differences. Descriptively explained that only the control group and group 1 did not

Table 1 Description of mean differences and standard deviations between groups with ESI scoring system

\begin{tabular}{cccc}
\hline \multirow{2}{*}{ Group } & \multicolumn{2}{c}{ Descriptive } & \\
\cline { 2 - 3 } Mean & SD & ANOVA \\
\hline Control & 1.0000 & 0.6324 & $\mathrm{~F}=$ \\
1 & 3.5000 & 0.5472 & 22.319 \\
2 & 2.8333 & 0.7527 & $\mathrm{P}=0.00$ \\
3 & 1.3333 & 0.5164 & $\alpha=0.05$ \\
\hline
\end{tabular}

Table 2 Description of the differences and standard deviations the number of cracks between groups and ANOVA test results

\begin{tabular}{cccc}
\hline \multirow{2}{*}{ Group } & \multicolumn{2}{c}{ Descriptive } & \multirow{2}{*}{ ANOVA } \\
\cline { 2 - 3 } & Mean & SD & \\
\hline Control & 1.33 & 1.033 & $\mathrm{~F}=42.973$ \\
1 & 1.33 & 1.516 & $\mathrm{P}=0.00$ \\
2 & 5.17 & 1.753 & $\alpha=0.05$ \\
3 & 2.83 & 1.753 & \\
\hline
\end{tabular}

\section{Scoring method}

Researcher used ESI to score the damage of the enamel surface and count the number and the length of the scratches. For statistic we used OneWay ANOVA parametric test because the $F$ value of all groups is greater than $\alpha(\mathrm{P}>0.05)$ it can be concluded that the data is normally distributed. ESI score varied in each group. From parametric statistical test. One-way ANOVA shows the highest significance value in group 1 , which is 0.883 ( $p>0.05$ ). The table above shows that the highest mean value is in group 1 , which is 3.5 , while the mean value in group 3 is 1.33 . The results of differences in the control group and group 1 showed a significance value of $0.00(p<0.05)$. In the comparison of the control group with group 2 shows the significance value of $0.01 \quad(\mathrm{p}<0.05)$, while the comparison in the control group compared to group 3 , shows the significance value of 0.832 ( $p>0.05)$.

Description number of cracks explain on table 1 and table 2 it is found that the group with the roughest surface was group 2 with average crack have significant differences. The control group and group 2 had a significant difference with $0.00(\mathrm{P}<0.05)$ and a significant difference between the control group and group 3 with a value of 0.04 ( $\mathrm{P}<0.05)$. For comparison between groups that were given treatment also showed a significant difference in group 2 with group 3 with a value of $0.04(\mathrm{P}<0.05)$.

Table 3 Description of the number of cracks differences between groups using the Schefe test

\begin{tabular}{cccc}
\hline \multirow{2}{*}{ Group } & \multicolumn{2}{c}{ Descriptive } & \\
\cline { 2 - 3 } Control & 0.24033 & 0.11829 & ANOVA \\
1 & 0.09483 & 0.14692 & $\mathrm{~F}=45.256$ \\
2 & 0.79800 & 0.11943 & $\mathrm{P}=0.00$ \\
3 & 0.39937 & 0.29510 & $\alpha=0.05$ \\
\hline
\end{tabular}

Table 4 Description of mean differences and standard deviations of the length of cracks between groups and ANOVA test results

\begin{tabular}{ccrcc}
\hline Group & Control & 1 & 2 & 3 \\
Control & - & 0.216 & $0.000^{*}$ & $0.030^{*}$ \\
1. & 0.216 & - & $0.000^{*}$ & $0.000^{*}$ \\
2. & $0.00^{*}$ & $0.00^{*}$ & - & $0.001^{*}$ \\
3. & $0.030^{*}$ & $0.000^{*}$ & $0.001^{*}$ & - \\
\hline
\end{tabular}

was 5.17 and the standard deviation was 1.753 . The groups with the smallest mean were groups 1 and 3 , in this group the mean value in each group was equal to 1.33. The standard deviation for group 1 was 1,033 and in group 3 was 1,516. ANOVA results were $0.00(\mathrm{P}<0.05)$ indicated that there were many differences in each group. Group 3 has a mean value of 2.83 with a standard deviation of 1.753. Table 3 describes the difference in values between groups and shows the difference in results between groups except in the control group and group 1. Group 1 has the same value as the control group.

Table 3 and table 4 shows descriptive results the length of the crack using Schefe test and continue with ANOVA. Descriptively explained that only the control group and group 1 did not have significant differences. The control group and group 2 had a significant difference with 0.00 (P $<0.05)$ and a significant difference between the control group and group 3 with a value of 0.04 (P $<0.05$ ). For comparison between groups that were given treatment also showed a significant 


\section{Indonesian Journal of Dental Medicine}

Volume 1 Issue 1 2018; 59-65

difference in group 2 with group 3 with a value of $0.04(\mathrm{P}<0.05)$.

\section{DISCUSSION}

According to Joanna, there is no adhesive cleaning technique without causing enamel surface damage. The reason underlying this opinion was the use of acid etching which results in the infiltration of resin into the enamel and decrease enamel hardness than any abrasive material. The effort taken by a clinician was to reduce and minimize the risk of losing the outer layer of enamel, because the outermost layer is the hardest and contains fluoride. Enamel surface must be as smooth as possible after debonding 9 .

The same color between remaining adhesive and enamel material, made cleaning procedure was not easy. Many patients complain the remaining adhesive material, the alternatives are often used are polishing burs such as tapered tungsten carbide burs (\# 1171 or \# 1172), Arkansas stone burs or suitable abrasive discs ${ }^{3}$. The results of observations using SEM showed that significant surface differences between tooth in group control group, group without cleaning procedure and with cleaning procedure after debonding. Cleaning the remaining adhesive material using both types of bur showed a significant difference both at the magnification of SEM 50x and magnification of SEM 500x. The study also showed that enamel damage was evident after the release of the bracket without residual adhesive cleaning. Observations showed in group 1 that the bracket release was not followed by residual adhesion.

Removal showing uneven surfaces, there was a pile of dental adhesive material which accumulated on the tooth surface with varying depths (figure 1). Samples in group 2 which clean the remaining adhesive using Arkansas stone showed abrasion in several areas and many long cracks with varied depth. In 500x magnification showed cracks and long streaks enamel surfaces in irregular direction. ESI shows an average value of 2.83 dominant in this group. In group 3 that used tungsten carbide bur showed a smoother surface, there were several fine scratches and still seemed to describe the morphological structure of the normal enamel surface. ESI shows that in this treatment group had the smoothest surface and good morphological structure of the enamel surface whose surface is close to the surface image of the control group (figure 1).

According to Grocholewicz, He did an analysis of 44 studies where there was a debate between cleaning techniques for residual adhesive using tungsten carbide bur with Arkansas stone. In this study no further work was discussed, but the results of SEM showed that the use of tungsten carbide bur to clean the remaining adhesive material showed the most delicate surface image compared to the use of greenstone, diamond bur and sharp band remover. While the use of Arkansas stone shows a rough SEM image and there is deep and irregular scratching ${ }^{10}$.

Bauman conducted a study to compare cleansing residual adhesive using tungsten carbide bur analyzed using EDI and visually observed using dental loupes showed that using tungsten carbide bur shows EDI scores 0 and 1 in the most samples and visual observations using dental loupes showed no significant differences in teeth before and after being treated. The statement of Grocholewicz and Bauman were match with the results of SEM in group 4. Visual observation in group 3 with $100 x$ magnification and $500 x$ in figure 1D showed a smooth enamel surface, there are no crack lines or material debris on the surface of the tooth. There is a picture resembling pores on the enamel surface due to the acid etching application that causes micro porosity on the enamel surface. Statistically the number of cracks and crack's lengths in groups 4 was lower than group 3. Using Arkansas stone left debris on enamel surface ${ }^{10,11}$.

Ryf argued that cleaning the rest of the adhesive material that is still attached intact to the surface of the tooth (bulky) using 12 flute tungsten carbide bur using contra angle high speed (above 200,000 $\mathrm{rpm}$ ) with an adequate water spray and water spray to maintain the heat. On adhesive residual cleaning, the increasing in temperature will stimulate intra pulpa pressure. Cooling procedures using water and air sprays is very important to prevent damage pulp ${ }^{12}$. Cleaning the remaining adhesive material with tungsten carbide bur using Contra angle high speed handpiece without water cooling will increase the temperature to the critical value of $5.58^{\circ} \mathrm{C}$ for pulp health. Cleaning the remaining adhesive material with water spray never reduce the critical temperature. Number of threads on tungsten carbide bur affects resin composite wear and heating during cleaning remnants of adhesive material from the enamel surface. A temperature rises of $9.4^{\circ} \mathrm{C}$ was found when using tungsten carbide bur 6-fluter, followed by 12 -fluted $\left(6.5^{\circ} \mathrm{C}\right)$ and $1.2^{\circ} \mathrm{C}$ tungsten carbide burs use tungsten carbide 40-fluted bur ${ }^{12}$.

Based on the research, it was found that cleaning adhesive residue after debonding was absolutely done to clean all material on the tooth surface, the remaining adhesive material would cause 
discoloration and retention of plaque on the teeth ${ }^{14}$. Meanwhile, according to the study of the proper cleaning of the residual adhesive material is using tungsten carbide bur, the more curvature the bur, the cleaning of the remaining adhesive is better and able to prevent the increase in temperature. Analyzing surface damage due to cleaning of residual adhesive material, must consider aspects of iatrogenic damage, the rotary instrument used for removal of residual residues can cause enamel abrasion in an amount that depends on the size and composition of the abrasive particles, rotational speed, and pressure on the enamel surface. Besides that, the final decisive factor is operator.

Based on the study of comparison of tungsten carbide and Arkansas stone for adhesive removal on enamel surface after debonding procedure it can be concluded that best method to clean the remaining adhesive is using 12-flutted tungsten carbide bur using contra angle high speed and with adequate water flow and sprays to prevent increasing of temperature that damage dental pup.

\section{REFERENCES}

1. Michael B Olsen. 2011. The Effect Of Phosphoric Acid Etch In Conjunction With Self-Etching Primer On Enamel Surfaces And Resultant Orthodontic Bracket Bond Strength. Thesis. Oraland Craniofacial Sciences Master Degree. University of MissouriKansas City

2. Hasegawa A, Ikeda I, Kawaguchi S. (2012) Color and Translucency of in Vivo Natural Central Inci sors. J Prosthet Dent; 83:41-3.

3. Neelutpal Bora., Nabanita Baruah. (2015) Debonding in Orthodontics: A Review. University $\mathbf{J}$ Dent Scie, No. 1, Vol. 3

4. Hosein I, Sherriff M, Ireland AJ. (2014) Enamel loss during bonding, debonding, and cleanup with use of a self-etching primer. Am J Orthod Dentofacial Orthop; 126: 717-24.

5. Oliver RG. (2003). The Effect of Different Methods of Bracket Removal on the Amount of Residual Adhesive. Am J Orthod Dentofacial Orthop. 93(3): 196-200.

6. Cardoso, L. A. M., Valdrighi, H. C., Vedovello Filho, M., \& Correr, A. B. (2014). Effect of adhesive remnant removal on enamel topography after bracket debonding. Dental Press Journal of Orthodontics, 19(6), 105-112.

7. Macieski, K., Rocha, R., Locks, A., \& Ribeiro, G.U. (2011). Effects evaluation of remaining resin removal (three modes) on enamel surface after bracket debonding. Dental Press J Orthod, 14616(5), 146-154.

8. Zachrisson B, Arthun J. (1980): Enamel Surface after Various Debonding Procedures. Am J Orthod: 1980:75 (2):12 1-137.

9. Joanna J.O., Tomasz S. (2014) Effect of Orthodontic Debonding and Adhesive Removal on the Enamel Current Knowledge and Future Perspectives - a Systematic Review, Med Sci Monit. 2014; 20: 1991 2001.

10. Grocholewicz, K. (2014). Effect of Orthodontic Debonding and Adhesive Removal on the Enamel - Current Knowledge and Future Perspectives - a Systematic Review. Medical Science Monitor, 20, 1991-2001.

11. Buonocore MG, Wileman WR, Brudevold F. (1960) A Simple Method of Increasing The Adhesion Of Acrylic Filling Materials To Enamel Surfaces. J Dent Res.; 33:694

12. Ryf, S., Flury, S., Palaniappan, S., Lussi, A., Van Meerbeek, B., \& Zimmerli, B. (2012). Enamel loss and adhesive remnants following bracket removal and various clean-up procedures in vitro. European Journal of Orthodontics, 34(1), 25-32.

13. Ahrari, F., Akbari, M., Akbari, J., \& Dabiri, G. (2013). Enamel surface roughness after debonding of orthodontic brackets and various clean-up techniques. Journal of Dentistry (Tehran, Iran), 10(1), 82-93. Retrieved

14. Kitayama, S., Nikaido, T., Ikeda, M., Foxton, R. M., \& Tagami, J. (2007). Enamel bonding of self- etch and phosphoric acid-etch orthodontic adhesive systems. Dental Materials Journal, 26(1), 135- 143. 